\title{
miR-205-5p inhibits cell migration and invasion in prostatic carcinoma by targeting ZEB1
}

\author{
LIANPENG $\mathrm{LI}^{1}$ and SHOUQIANG $\mathrm{LI}^{2}$ \\ ${ }^{1}$ Department of Urological Surgery, Binzhou Central Hospital, Binzhou, Shandong 251700; \\ ${ }^{2}$ Department of Urological Surgery, People's Hospital of Dongying District, Dongying, Shandong 257000, P.R. China
}

Received November 29, 2017; Accepted May 16, 2018

DOI: $10.3892 / \mathrm{ol} .2018 .8862$

\begin{abstract}
Many studies have demonstrated that miRNAs have influence on tumorigenesis and progression of human cancers, including invasion and migration. Thus, the role of miR-205/ZEB1 axis for the migration and invasion of prostate cancer cells was explored in the present study. The miR-205-5p and zinc finger E-box binding homeobox 1 (ZEB1) mRNA expression levels were observed in prostate cancer tissues or cell lines via reverse transcription-quantitative PCR (RT-qPCR), and the protein level of ZEB1 was measured by western blotting. Dual-Luciferase Reporter Assay was used to verify the relationship between miR-205-5p and ZEB1. In addition, cell migration and invasion was measured by Transwell assay. The results revealed that, compared with the control, downregulation of miR-205-5p was detected in prostate cancer tissues and cell lines, and miR-205-5p overexpression was found to inhibit cell migration and invasion. Moreover, miR-205-5p was confirmed to directly target ZEB1 in prostate cancer. Importantly, ZEB1 was identified to weaken the inhibitory effect of miR-205-5p in prostate cancer. In conclusion, miR-205-5p inhibited cell migration and invasion in prostatic carcinoma by targeting ZEB1 and miR-205-5p/ZEB1 axis shows potential to be developed in therapeutic strategies for prostate cancer.
\end{abstract}

\section{Introduction}

Prostate cancer is one of the most common male malignancies, with a mortality rate of about 300,000 people every year worldwide (1). Although advanced therapeutic techniques are widely used for patients with prostate cancer, local recurrence frequently occurs after treatment. Moreover, dysregulation of oncogene or tumor suppressor factors has been demonstrated to be closely associated with the development and progression

Correspondence to: Dr Lianpeng Li, Department of Urological Surgery, Binzhou Central Hospital, 108 Huancheng South Road, Huimin, Binzhou, Shandong 251700, P.R. China

E-mail: vxo174817@163.com

Key words: prostatic carcinoma, miR-205-5p, invasion, migration, zinc finger E-box binding homeobox 1 of human cancers (2). Therefore, it is necessary to better understand the molecular mechanism of prostate cancer which may facilitate the development of new therapeutic techniques for human cancers.

Recently, microRNAs were reported to repress the expression of corresponding genes through binding with their 3'-UTR of mRNA which led to mRNA degradation or inhibition of protein translation (3). Through regulating the expression of target genes, miRNA plays an important role in various cell biological activities, including cell survival, proliferation, differentiation, and apoptosis (4). Moreover, many miRNAs have been reported to associate with the occurrence and development of human cancers including prostate cancer (5-8). For example, miR-199a-3p was found to suppress prostate cancer cell proliferation and invasion by targeting SMAD1 (9) while miR-483-5p promoted them by targeting RBM5 (10). Especially, as a tumor suppressor, miR-205 has been identified in many human cancers. For instance, miR-205 suppressed proliferation and invasion by targeting IGF1R in human cervical cancer (11). miR-205 acted as a biological marker in non-small cell lung cancer (12). More importantly, miR-205 was demonstrated to be downregulated and regulating $\mathrm{Bcl} 2$ expression in prostate cancer (13). However, the special role of miR-205-5p is still to be investigated in prostate cancer.

Additionally, zinc finger E-box binding homeobox 1 (ZEB1) belonging to zinc finger transcription factor superfamily was found to be involved in regulating transcription (14). Many studies have reported the function of ZEB1 in the occurrence of human cancers including osteosarcoma and cervical cancer $(15,16)$. Moreover, ZEB1 was upregulated and had a promoting role in prostate cancer (17), and it was reported that miR-128 modulated cell chemosensitivity and invasion in prostate cancer by regulating ZEB1 expression (18). However, the regulatory mechanism of miR-205/ZEB1 axis in prostate cancer is still unknown.

Therefore, the purpose of this study was to explore the regulatory mechanism of miR-205-5p/ZEB1 axis in prostate cancer, and the findings might provide original biomarkers to diagnose prostate cancer at early stage.

\section{Materials and methods}

Tissue samples. Eighteen pairs of prostate tissues and adjacent normal prostate tissues were collected from Binzhou City 
Center Hospital (Binzhou, China) between January 2015, and May 2017. The adjacent normal tissues were representative of tissues that were located $2-5 \mathrm{~cm}$ away from tumors that were confirmed to contain no cancer cells. All tissue specimens were immediately snap-frozen in liquid nitrogen and then stored at $-80^{\circ} \mathrm{C}$ for a reverse transcription-quantitative PCR (RT-qPCR) test. Before the surgery, none of prostate patients received any other treatments. Moreover, written informed consent was obtained from all patients or guardians, and this experiment was approved by the Institutional Ethics Committee of Binzhou City Center Hospital (Binzhou, China).

Cell culture. Human PCa cell lines LNCaP, DU-145 cell lines and the normal prostate epithelial cell line RWPE-1 were obtained from the Cell Bank of Institute of Biochemistry and Cell Biology (Shanghai, China). In addition, 293T cell line was cultured to perform the luciferase reporter assay. These cells were cultured in RPMI-1640 (Gibco: Thermo Fisher Scientific, Inc., Carlsbad, CA, USA) supplemented with $10 \%$ fetal bovine serum (FBS) in an atmosphere of $5 \% \mathrm{CO}_{2}$ at $37^{\circ} \mathrm{C}$.

$R T$-qPCR. TRIzol reagent (Invitrogen: Thermo Fisher Scientific, Inc., Carlsbad, CA, USA) was applied to extract total RNA in tissue samples and cell lines. RT-qPCR was conducted using the SYBR-Green PCR Master Mix on an ABI 7300HT RT-PCR system (Applied Biosystems: Thermo Fisher Scientific, Inc., Foster City, CA, USA) to detect expression of miR-205-5p and ZEB1 mRNA. The reaction conditions were $95^{\circ} \mathrm{C}$ for $3 \mathrm{~min}$, and 40 cycles of denaturation at $95^{\circ} \mathrm{C}$ for $15 \mathrm{sec}$ and annealing/elongation at $58^{\circ} \mathrm{C}$ for $30 \mathrm{sec}$. The miR-205 forward, 5'-TCC TTC ATT CCA CCG GAG TCT G-3' and reverse, 5'-GCG AGC ACA GAA TTA ATA CGA C-3'; U6 forward, 5'-ATT GGA ACG ATA CAG AGA AGA TT-3' and reverse, 5'-GGA ACG CTT CAC GAA TTT G-3'; ZEB1 forward, 5'-CAG GCA GAT GAA GCA GGA TG-3' and reverse, 5'-CAG CAG TGT CTT GTT GTT GTA G-3'; and GAPDH forward, 5'-TTG GTA TCG TGG AAG GAC TCA-3' and reverse, 5'-TGT CAT CAT ATT TGG CAG GTT-3'. U6 or GAPDH served as control for miR-205-5p or ZEB1. The miR-205-5p or ZEB1 expression levels were analyzed using the $2^{-\Delta \Delta C q} \operatorname{method}(19)$.

Cell transfection. The miR-205-5p mimic (HMI0359-5NMOL) and miR-205-5p inhibitor (HLTUD0359) were obtained from Sigma-Aldrich: Merck KGaA (Shanghai, China). The on-target plus siRNA that targets human ZEB1 was purchased from GE Healthcare Dharmacon, Inc. (J-006564-10-0005; Lafayette, CO, USA). Then they were transferred into prostate cells respectively with Lipofectamine 2000 (Invitrogen: Thermo Fisher Scientific, Inc.) according to manufacturers' instructions.

Western blotting. The protein samples were obtained using RIPA buffer. Proteins were separated through $10 \%$ SDS-PAGE and then incubated with 5\% non-fat milk blocked polyvinylidene fluoride (PVDF) membranes (EMD Millipore, Billerica, MA, USA). Next we incubated the membranes overnight at $4^{\circ} \mathrm{C}$ with anti-ZEB1 (dilution 1:1,000; rabbit monoclonal; ab203829; Abcam, Cambridge, MA, USA), anti-GAPDH (dilution 1:1,000; mouse monoclonal;
60004-1-Ig; Wuhan Sanying Biotechnology, Wuhan, China) and subsequently incubated with goat anti-rabbit $\operatorname{IgG}$ H\&L (HRP) (dilution 1:3,000; ab6721; Abcam) secondary antibodies. Then, protein expression levels were measured by increasing the chemiluminescence and exposure to chemiluminescent film (Pierce: Thermo Fisher Scientific, Inc., Shanghai, China).

Luciferase reporter assay. TargetScan (http://www.targetscan. org/) predicts biological targets of miRNAs by searching for the presence of conserved $8 \mathrm{mer}$, $7 \mathrm{mer}$, and $6 \mathrm{mer}$ sites that match the seed region of each miRNA (20). The ZEB1 was predicted as a taret gene of miR-205-5p. Then the wild-type and mutated 3'-UTR sequences of ZEB1 mRNA containing the miR-205-5p targeting sequence were inserted into the pGL3 basic plasmid (GenScript, Nanjing, China) for luciferase reporter experiments. Then, the plasmid and miR-205-5p mimic were transfected into cells using Lipofectamine 2000 (Invitrogen: Thermo Fisher Scientific, Inc.). Finally, Dual-Luciferase Reporter Assay system (Beyotime Institute of Biotechnology, Beijing, China) was applied to measure luciferase activity.

Transwell assay. Cell migration was assessed using uncoated Transwell membrane (BD Biosciences, Franklin Lakes, NJ, USA) with a pore size of $8 \mu \mathrm{m}$. The lower chamber was filled with complete medium while the upper chamber was filled with serum-free medium containing $1 \times 10^{5}$ prostate cells. These cells were cultured at $37^{\circ} \mathrm{C}$ for $48 \mathrm{~h}$ and then fixed with methanol. By using cotton swabs, non-migrating cells on the upper membranes were carefully removed. At the same time, the migration cells on the lower membrane were stained by $0.1 \%$ crystal violet and photographed under an inverted light microscope (Zeiss, Oberkochen, Germany). The invasion test was carried out according to the method of migration assay except for the pre-coated membrane.

Statistical analysis. All statistical analysis was conducted using SPSS 17.0 (SPSS, Inc., Chicago, IL, USA) and GraphPad Prism 6.0 software (GraphPad Software, Inc., La Jolla, CA, USA). Data are presented as mean \pm SD. The differences between groups were examined by analysis of variance (ANOVA) with Tukey-Kramer post hoc test and Student's $\mathrm{t}$-tests. Pearson's correlation test was employed to evaluate the association between miR-205-5p and ZEB1 mRNA expression. It was considered significant at $\mathrm{P}<0.05$.

\section{Results}

miR-205-5p is downregulated in prostate tissues and cell lines. Firstly, miR-205-5p expression level was examined in prostate tissues and cell lines using RT-qPCR assay. The results showed that miR-205-5p expression was lower in prostate cancer tissue than the normal prostate tissues $(\mathrm{P}<0.01$, Fig. 1A). Moreover, the expression levels of miR-205-5p in the DU-145 and LNCaP cells were significantly reduced compared with the normal prostate cells RWPE-1 ( $\mathrm{P}<0.01$, Fig. 1B). All these results indicated that miR-205-5p was downregulated in prostate cancer.

Cell migration and invasion are inhibited by miR-205-5p overexpression. In order to explore the function of 

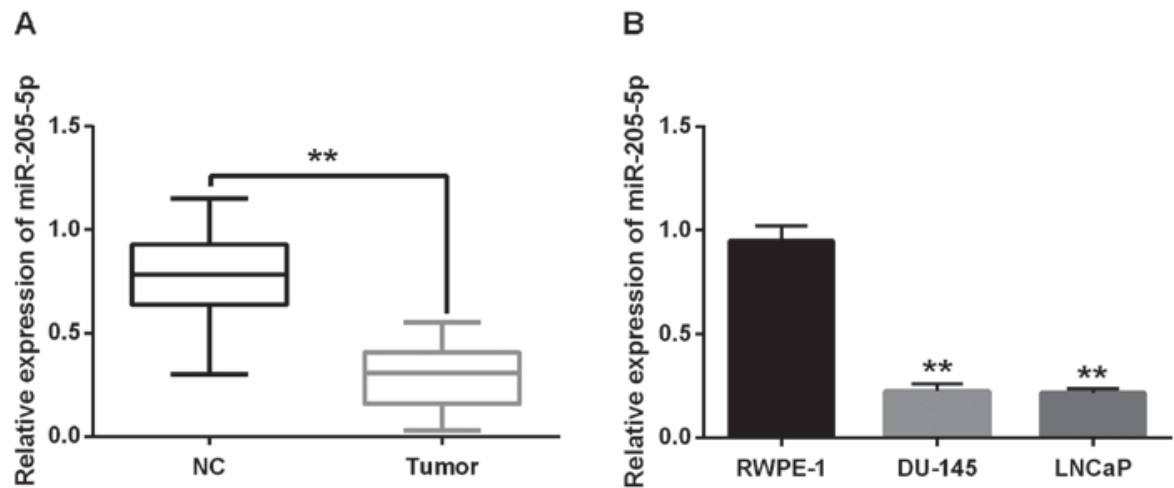

Figure 1. miR-205-5p expression is downregulated in prostatic carcinoma tissues and cell lines. (A) The miR-205-5p expression in prostatic carcinoma tissues was examined by RT-qPCR. (B) The miR-205-5p expression level in prostatic carcinoma cell lines (DU-145 and LNCaP) was detected by RT-qPCR. Transcription levels were normalized to U6 expression. ${ }^{* *} \mathrm{P}<0.01$. RT-qPCR, reverse transcription quantitative PCR.

A

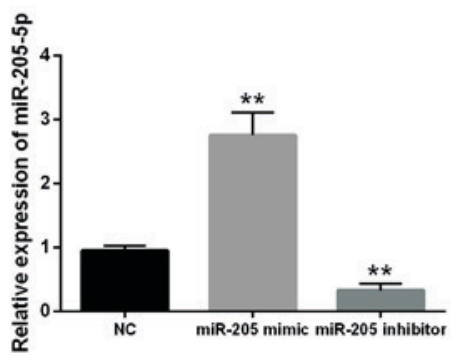

B
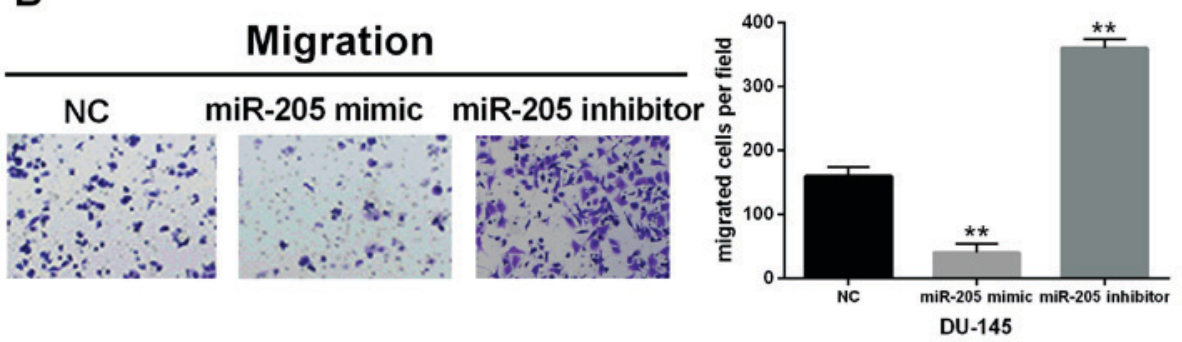

C
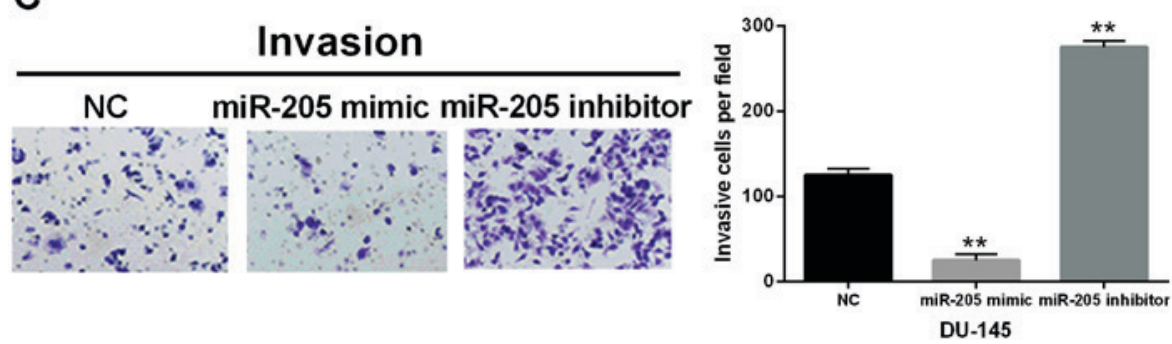

Figure 2. Overexpression of miR-205-5p inhibits cell migration and invasion in prostatic carcinoma. (A) The miR-205-5p expression in DU-145 cells containing miR-205-5p mimic or inhibitor was detected by RT-qPCR. (B and C) Transwell assays were performed to measure cell migration and invasion. ${ }^{* *} \mathrm{P}<0.01$. RT-qPCR, reverse transcription quantitative PCR.

miR-205-5p in human prostate cells, the miR-205-5p mimic or inhibitor was transfected into DU-145 cells, respectively. The RT-qPCR analysis suggested that the expression level of miR-205-5p in DU-145 cells with miR-205-5p mimic was obviously increased while decreased by miR-205-5p inhibitor $(\mathrm{P}<0.01$, Fig. 2A). Furthermore, the migration and invasion of prostate cancer cells were detected also in our study. The Transwell assay indicated that compared with the control group, miR-205-5p mimic reduced the migrated or invasive cell number to $\sim 25$ or $20 \%$, respectively. On the contrary, the migrated or invasive cell number in transfected cells with miR-205-5p inhibitor was 2.25 or 2.2 times that of the control group. The results implied that the migration and invasion of DU-145 cells were significantly inhibited by miR-205-5p overexpression $(\mathrm{P}<0.01)$ while markedly promoted by miR-205-5p silence compared with the control 


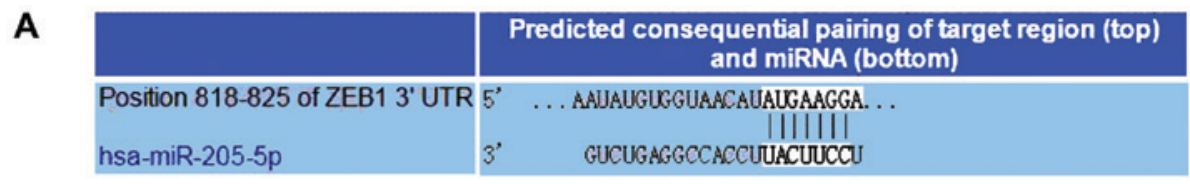

B

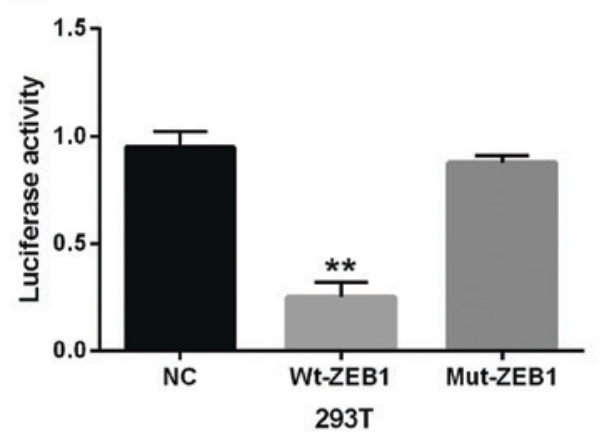

D

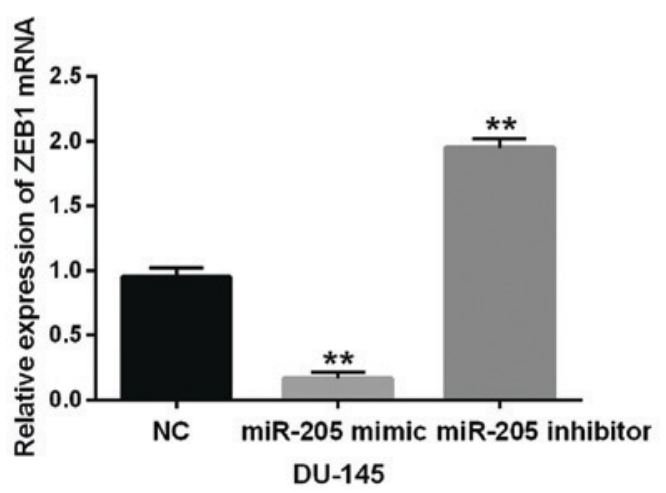

C

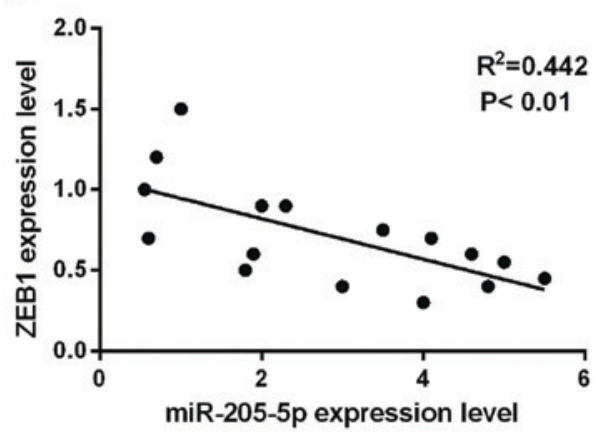

E

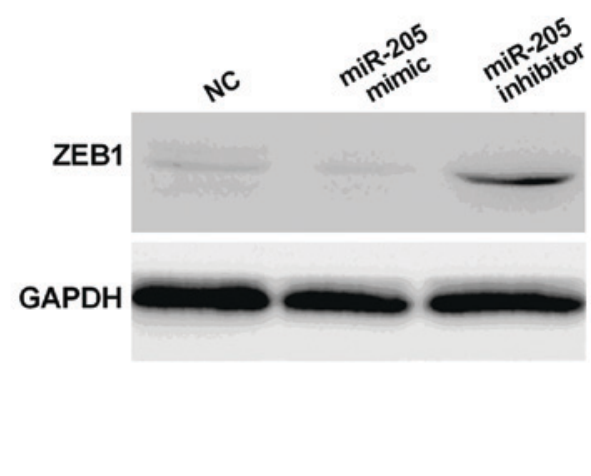

Figure 3. ZEB1 is a direct target gene of miR-205-5p. (A) The binding site of miR-205-5p bound with the 3'-UTR of the ZEB1 mRNA. (B) Luciferase assay. (C) The associateion between miR-205-5p and ZEB1 was identified. (D and E) The mRNA and protein expression of ZEB1 in cells containing miR-205-5p mimic or inhibitor. ${ }^{* *} \mathrm{P}<0.01$. ZEB1, zinc finger E-box binding homeobox 1.

group $(\mathrm{P}<0.01)$ (Fig. 2B and $\mathrm{C})$. Therefore, we deduced that miR-205-5p inhibited cell migration and invasion in prostate cancer.

ZEB1 is a direct target gene of miR-205-5p in prostatic carcinoma. The prediction of TargetScan (http://www.targetscan. org/) is shown in Fig. 3A indicating the binding site of miR-205-5p bound with the 3'-UTR of the ZEB1 mRNA. In order to confirm the above prediction, the ZEB1-wt or ZEB1-mut vector with miR-205-5p mimic was transfected into $293 \mathrm{~T}$ cells. Then the luciferase activity was detected to confirm the above result. As shown in Fig. 3B, miR-205-5p mimic reduced the luciferase activity of wild-type of ZEB1 by binding with its 3'-UTR $(\mathrm{P}<0.01)$ while almost no difference was found in cells co-transfected with ZEB1-mut and miR-205-5p mimic. In order to further confirm the relationship between miR-205-5p and ZEB1, we analyzed the association between miR-205-5p and ZEB1 mRNA expression using Pearson's correlation test, and we found that ZEB1 was negatively correlated with miR-205-5p ( $\mathrm{P}<0.01$, Fig. 3C). Besides, the expression of mRNA and protein of ZEB1 was clearly decreased by the miR-205-5p mimics $(\mathrm{P}<0.01)$ while increased by the miR-205-5p inhibitor compared with the control group $(\mathrm{P}<0.01$, Fig. 3D and $\mathrm{E})$. It indicated that miR-205-5p could inhibit the expression of ZEB1 by targeting its
3'-UTR. In conclusion, these results mainly revealed that ZEB1 was a direct target gene of miR-205-5p in prostatic carcinoma and had negative association with miR-205-5p.

ZEB1 knockdown inhibits cell migration and invasion. ZEB1 was found to be upregulated in DU-145 and LNCaP cell lines (Fig. 4A). Then, siRNA-ZEB1 was used to inhibit ZEB1 expression to investigate its effect in prostate cancer cells (Fig. 4B). The Transwell assay indicated that compared with the control group, siRNA-ZEB1 reduced the migrated or invasive cells number to $\sim 38$ or $25 \%$, respectively. It indicated that the cell migration and invasion were remarkably decreased by ZEB1 silence in prostate cancer (Fig. 4C and D). We inferred that ZEB1 knockdown inhibited cell migration and invasion in prostate cancer.

Cell migration and invasion are affected by dysregulation of miR-205-5p via targeting ZEB1. In order to further confirm the relationship between the miR-205-5p and ZEB1 in prostate cancer, ZEB1 plasmid and miR-205-5p mimic were transfected into DU-145 cells. Then the expression level of ZEB1 in these transfected cells was examined by RT-qPCR and western blotting assay. Simultaneously, the cell migration and invasion were detected. We found that ZEB1 expression was declined in 
A

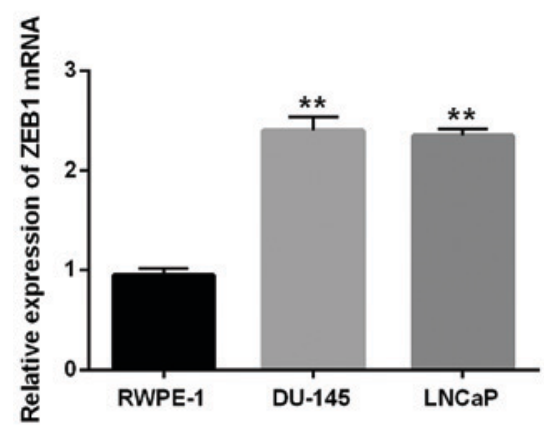

C

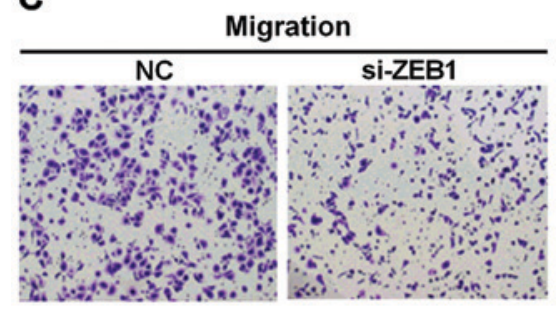

D

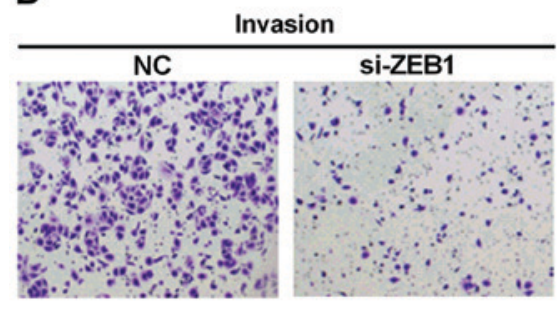

B
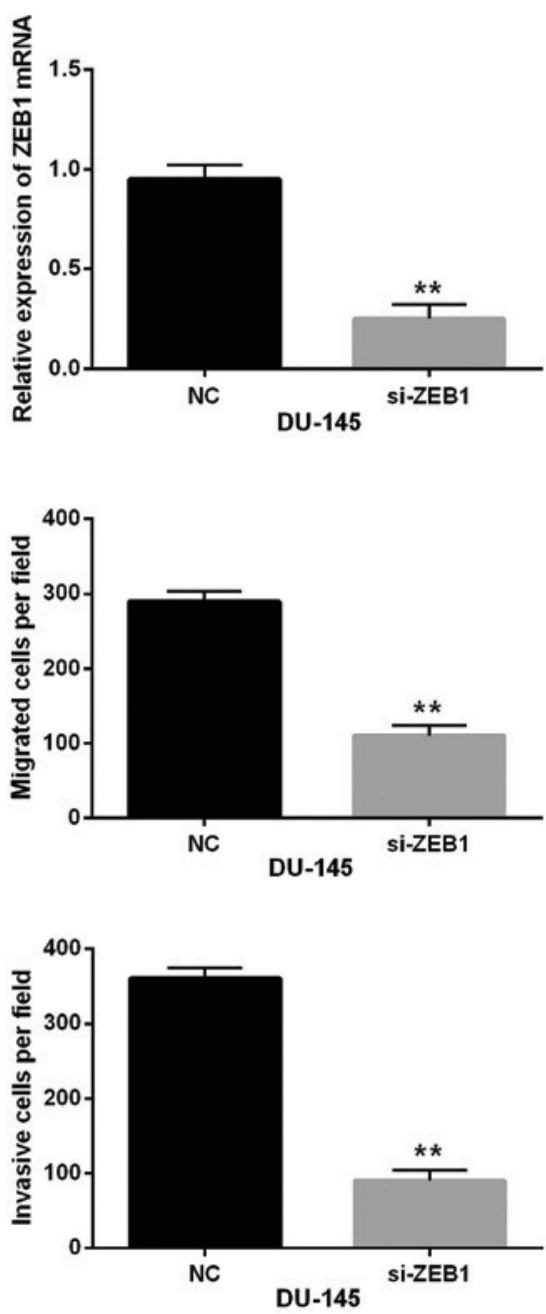

Figure 4. ZEB1 knockdown inhibits cell migration and invasion. (A) The ZEB1 expression in prostatic carcinoma cell lines (DU-145 and LNCaP). (B) The expression of ZEB1 was examined by RT-qPCR in cells containing siRNA-ZEB1. (C and D) The cell migration and invasion in prostatic carcinoma cells transfected with siRNA-ZEB1 ( ${ }^{* *}$ P $\left.<0.01\right)$. ZEB1, zinc finger E-box binding homeobox 1; RT-qPCR, reverse transcription quantitative PCR.

cells containing miR-205-5p mimic, whereas little change for ZEB1 expression was found in cells with ZEB1 plasmid and miR-205-5p mimic compared with the control group (Fig. 5A). Moreover, western blotting assay confirmed the above result again (Fig. 5B). Importantly, ZEB1 remarkably weakened the suppressive effect of miR-205-5p on cell migration and invasion in prostate cancer (Fig. 5C and D). We considered that ZEB1 could reverse the effect of miR-205-5p in prostate cancer.

\section{Discussion}

In the present study, the downregulation of miR-205-5p was identified in the human prostate cancer, and miR-205-5p inhibited cell migration and invasion in prostatic carcinoma by targeting ZEB1. It was demonstrated that miR-205 has a different effect in different human cancers. Some studies demonstrated that miR-205 had a carcinogenic effect in he occurrence of tumors $(21,22)$. On the contrary, downregulation of miR-205 was also detected in many cancers acting as a tumor suppressor in previous studies $(11,13,23)$. These results were consistent with our results that miR-205 expression was decreased in prostate cancer. Thus, miR-205 may act as an indicator for the diagnosis of prostate cancer patients.

Moreover, miR-205 was reported to regulate cell proliferation, migration and invasion in all kinds of human cancers $(24,25)$. In this research, miR-205-5p overexpression suppressed the migration and invasion of prostate cancer cells. On the contrary, Nie et al demonstrated that miR-205 overexpression promoted the proliferation, migration and invasion in nasopharyngeal carcinoma (26). These findings suggested that different effects of miR-205 on tumor pathogenesis depend on different human cancers.

Additionally, miR-205 was found to bind to the 3'-UTR of ZEB1 to degrade its mRNA and inhibit the translation of proteins. Increased evidence indicated that ZEB1 was strongly associated with the biological behavior of the tumor, such as migration and invasion, which greatly facilitated the formation and development of tumors $(27,28)$. Moreover, many miRNAs have been reported to be involved in ZEB1 regulation. For instance, ZEB1 promoted cell metastasis and invasion in ESCC regulatd by miR-125a/miR-99b/let-7e (29). miR-429 suppressed ZEB1 expression to regulate the progression and metastasis 
A

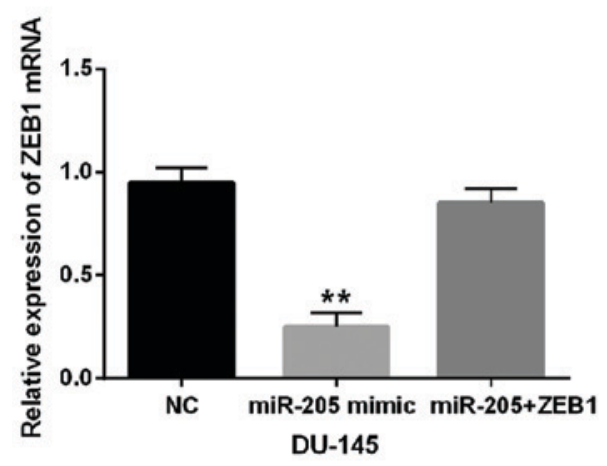

C

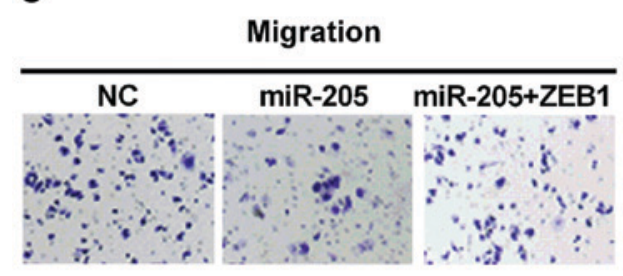

D

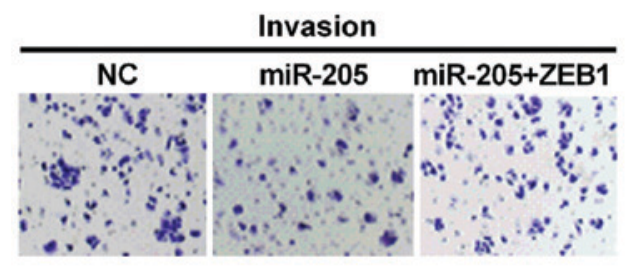

B
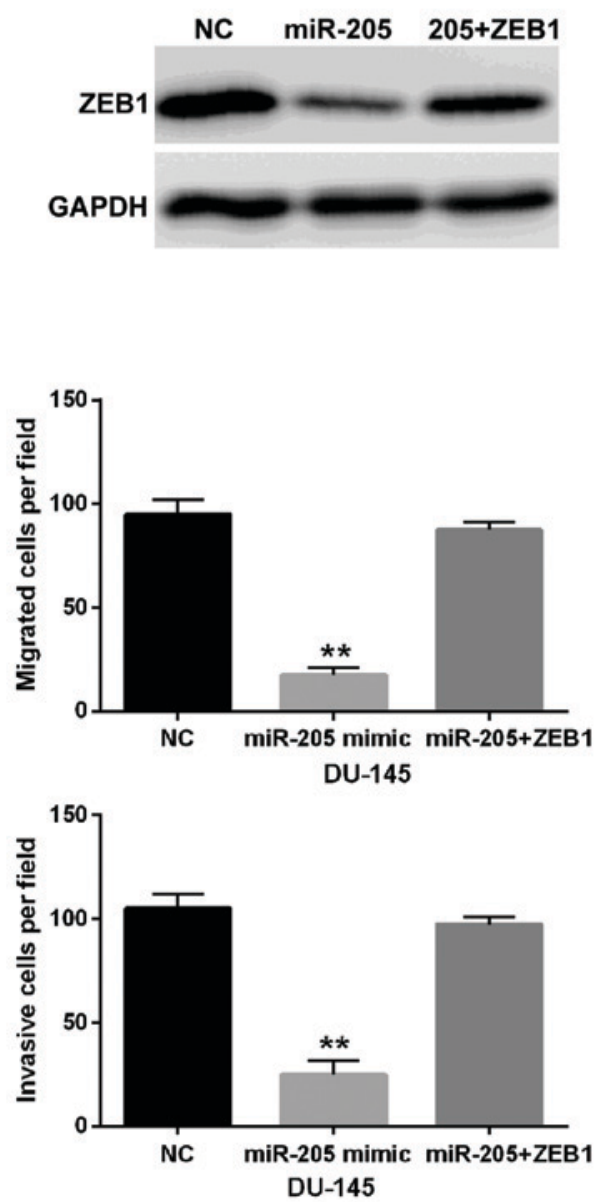

Figure 5. Cell migration and invasion are affected by dysregulation of miR-205-5p via targeting ZEB1. (A) Expression levels of ZEB1 were examined by RT-qPCR after transfection of miR-205-5p mimic and ZEB1 vector. (B) Expression levels of ZEB1 were examined by western blotting after transfection of mimic and ZEB1 vector. (C and D) The cell migration and invasion in prostatic carcinoma cells transfected with mimic and ZEB1 vector. ${ }^{* *} \mathrm{P}<0.01$. ZEB1, zinc finger E-box binding homeobox 1; RT-qPCR, reverse transcription quantitative PCR.

of osteosarcoma (30). In current research, miR-205-5p was also found to repress ZEB1 expression, and ZEB1 was found to promote the migration and invasion of prostate cancer cells. Importantly, ZEB1 was also identified to weaken the suppressive effect of miR-205-5p in prostate cancer. Thus, we considered that miR-205-5p inhibited cell migration and invasion in prostatic carcinoma by suppressing ZEB1 expression.

In summary, miR-205-5p downregulation and ZEB1 upregulation was identified in prostate cancer. Moreover, miR-205-5p suppressed cell migration and invasion of prostatic carcinoma through inhibiting ZEB1 expression. All the results suggested that the miR-205-5p/ZEB1 axis has potential to contribute to the treatment of prostate cancer.

\section{Acknowledgements}

Not applicable.

\section{Funding}

No funding was received.

\section{Availability of data and materials}

The datasets used and/or analyzed during the present study are available from the corresponding author on reasonable request.

\section{Authors' contributions}

LL contributed to the design and conception of the study, the data collection and analysis, and wrote the manuscript. SL also contributed to the conception of the study. Both authors have read and approved the final manuscript.

\section{Ethics approval and consent to participate}

The study was approved by the Ethics Committee of Binzhou City Center Hospital (Binzhou, China). Signed informed consents were obtained from the patients or guardians.

\section{Patient consent for publication}

Not applicable. 


\section{Competing interests}

The authors declare that they have no competing interests.

\section{References}

1. Tao ZQ, Shi AM, Wang KX and Zhang WD: Epidemiology of prostate cancer: Current status. Eur Rev Med Pharmacol Sci 19: 805-812, 2015.

2. Debebe $\mathrm{Z}$ and Rathmell WK: Ror2 as a therapeutic target in cancer. Pharmacol Ther 150: 143-148, 2015.

3. Croce CM and Calin GA: miRNAs, cancer, and stem cell division. Cell 122: 6-7, 2005

4. Liu X, Fortin K and Mourelatos Z: MicroRNAs: Biogenesis and molecular functions. Brain Pathol 18: 113-121, 2008

5. Zhao N, Lin T, Zhao C, Zhao S, Zhou S and Li Y: MicroRNA-588 is upregulated in human prostate cancer with prognostic and functional implications. J Cell Biochem: Oct 5, 2017 (Epub ahead of print)

6. Zhou YJ, Yang HQ, Xia W, Cui L, Xu RF, Lu H, Xue Z, Zhang B, Tian ZN, Cao YJ, et al: Down-regulation of miR-605 promotes the proliferation and invasion of prostate cancer cells by up-regulating EN2. Life Sci 190: 7-14, 2017.

7. Song XL, Tang Y, Lei XH, Zhao SC and Wu ZQ: miR-618 inhibits prostate cancer migration and invasion by targeting FOXP2. J Cancer 8: 2501-2510, 2017.

8. Luo B, Kang N, Chen Y, Liu L and Zhang Y: Oncogene miR-106a promotes proliferation and metastasis of prostate cancer cells by directly targeting PTEN in vivo and in vitro. Minerva Med 109: 24-30, 2018.

9. Qu F, Zheng J, Gan W, Lian H, He H, Li W, Yuan T, Yang Y, Li X, Ji C, et al: MiR-199a-3p suppresses proliferation and invasion of prostate cancer cells by targeting Smad1. Oncotarget 8: 52465-52473, 2017

10. Yang ZG, Ma XD, He ZH and Guo YX: miR-483-5p promotes prostate cancer cell proliferation and invasion by targeting RBM5. Int Braz J Urol 43: 1060-1067, 2017.

11. Pang H and Yue X: MiR-205 serves as a prognostic factor and suppresses proliferation and invasion by targeting insulin-like growth factor receptor 1 in human cervical cancer. Tumour Biol 39: 1010428317701308, 2017.

12. Duan B, Guo T, Sun H, Cai R, Rui Q and Xi Z: miR-205 as a biological marker in non-small cell lung cancer. Biomed Pharmacother 91: 823-830, 2017.

13. Verdoodt B, Neid M, Vogt M, Kuhn V, Liffers ST, Palisaar RJ, Noldus J, Tannapfel A and Mirmohammadsadegh A: MicroRNA-205, a novel regulator of the anti-apoptotic protein $\mathrm{Bcl} 2$, is downregulated in prostate cancer. Int J Oncol 43: 307-314, 2013

14. Pazos MC, Abramovich D, Bechis A, Accialini P, Parborell F, Tesone $\mathrm{M}$ and Irusta G: Gamma secretase inhibitor impairs epithelial-to-mesenchymal transition induced by TGF- $\beta$ in ovarian tumor cell lines. Mol Cell Endocrinol 440: 125-137, 2017.

15. Jiang R, Zhang C, Liu G, Gu R and Wu H: MicroRNA-126 inhibits proliferation, migration, invasion and EMT in osteosarcoma by targeting ZEB1. J Cell Biochem 118: 3765-3774, 2017.
16. Hu Y, Xie H, Liu Y, Liu W, Liu M and Tang H: miR-484 suppresses proliferation and epithelial-mesenchymal transition by targeting ZEB1 and SMAD2 in cervical cancer cells. Cancer Cell Int 17: 36, 2017

17. Cha YJ, Lee JH, Han HH, Kim BG, Kang S, Choi YD and Cho NH: MicroRNA alteration and putative target genes in high-grade prostatic intraepithelial neoplasia and prostate cancer: STAT3 and ZEB1 are upregulated during prostate carcinogenesis. Prostate 76: 937-947, 2016.

18. Sun X, Li Y, Yu J, Pei H, Luo P and Zhang J: miR-128 modulates chemosensitivity and invasion of prostate cancer cells through targeting ZEB1. Jpn J Clin Oncol 45: 474-482, 2015.

19. Livak KJ and Schmittgen TD: Analysis of relative gene expression data using real-time quantitative PCR and the 2(-Delta Delta C(T)) method. Methods 25: 402-408, 2001.

20. Volinia S, Visone R, Galasso M, Rossi E and Croce CM Identification of microRNA activity by Targets' Reverse EXpression. Bioinformatics 26: 91-97, 2010.

21. Wei J, Zhang L, Li J, Zhu S, Tai M, Mason CW, Chapman JA, Reynolds EA, Weiner CP and Zhou HH: MicroRNA-205 promotes cell invasion by repressing TCF21 in human ovarian cancer. J Ovarian Res 10: 33, 2017.

22. Niu K, Shen W, Zhang Y, Zhao Y and Lu Y: MiR-205 promotes motility of ovarian cancer cells via targeting ZEB1. Gene 574: 330-336, 2015

23. Zhang C, Long F, Wan J, Hu Y and He H: MicroRNA-205 acts as a tumor suppressor in osteosarcoma via targeting RUNX2. Oncol Rep 35: 3275-3284, 2016.

24. Chen S, Jin L, Nie S, Han L, Lu N and Zhou Y: MiR-205 inhibits growth and invasion of neuroblastoma by targeting cAMP responsive element binding protein 1. Oncol Res: Jun 26, 2017 (Epub ahead of print).

25. Zhuo $\mathrm{Z}$ and $\mathrm{Yu} \mathrm{H}$ : miR-205 inhibits cell growth by targeting AKT-mTOR signaling in progesterone-resistant endometrial cancer Ishikawa cells. Oncotarget 8: 28042-28051, 2017.

26. Nie G, Duan H, Li X, Yu Z, Luo L, Lu R, Ji Z and Zhang W: MicroRNA-205 promotes the tumorigenesis of nasopharyngeal carcinoma through targeting tumor protein p53-inducible nuclear protein 1. Mol Med Rep 12: 5715-5722, 2015

27. Dou J, He X, Liu Y, Wang Y, Zhao F, Wang X, Chen D, Shi F and Wang J: Effect of downregulation of ZEB1 on vimentin expression, tumour migration and tumourigenicity of melanoma B16F10 cells and CSCs. Cell Biol Int 38: 452-461, 2014

28. Wang Y, Yan S, Liu X, Zhang W, Li Y, Dong R, Zhang Q, Yang Q, Yuan C, Shen K, et al: miR-1236-3p represses the cell migration and invasion abilities by targeting ZEB1 in high-grade serous ovarian carcinoma. Oncol Rep 31: 1905-1910, 2014.

29. Ma J, Zhan Y, Xu Z, Li Y, Luo A, Ding F, Cao X, Chen H and Liu Z: ZEB1 induced miR-99b/let-7e/miR-125a cluster promotes invasion and metastasis in esophageal squamous cell carcinoma. Cancer Lett 398: 37-45, 2017.

30. Deng Y, Luan F, Zeng L, Zhang Y and Ma K: MiR-429 suppresses the progression and metastasis of osteosarcoma by targeting ZEB1. EXCLI J 16: 618-627, 2017.

This work is licensed under a Creative Commons Attribution-NonCommercial-NoDerivatives 4.0 International (CC BY-NC-ND 4.0) License. 